\title{
Erratum to: Hierarchies of difference boundary value problems
}

\author{
Sonja Currie* and Anne D Love
}

\section{"Correspondence:}

Sonja.Currie@wits.ac.za

School of Mathematics, University of the Witwatersrand, Private Bag 3, WITS 2050, Johannesburg, South Africa

\section{Erratum to: Boundary value problems, Volume 2011, Article ID 743135}

(1) The following paragraph needs to be inserted immediately after Theorem 4.2: It is important to note that the spectral parameter in the original boundary value problems given in cases (1)-(9) of Table 1 for Theorem 4.2 must first, without loss of generality, be shifted so as to ensure that all the eigenvalues are greater than zero. Similarly, for cases (10)-(12) of Table 1 for Theorem 4.2, the spectral parameter must be shifted so that the original boundary value problem has the least eigenvalue 0 . Having made these shifts we then take $z(n)$ to be a solution to (1.1) for $\lambda=\lambda_{0}=0$, i.e., throughout the paper we set $\lambda_{0}=0$.

(2) In Corollary 4.4 and its proof, there were typographical errors as well as notation that was not apparent. These should read as follows:

Corollary 4.4 If $\lambda_{1}, \ldots, \lambda_{s+l+m+1}$ are the eigenvalues of any one of the original boundary value problems (1)-(9), in Theorem 4.2, with corresponding eigenfunctions $u_{1}(n), \ldots$, $u_{s+l+m+1}(n)$, then

(i) $\lambda_{0}=0, \lambda_{1}, \ldots, \lambda_{s+l+m+1}$ are the eigenvalues of the corresponding transformed boundary value problems (1)-(3), in Theorem 4.2, with corresponding eigenfunctions $\frac{1}{z(n-1) c(n-1)}, u_{1}(n), \ldots, u_{s+l+m+1}(n)$;

(ii) $\lambda_{1}, \ldots, \lambda_{s+l+m+1}$ are the eigenvalues of the corresponding transformed boundary value problems (4)-(9), in Theorem 4.2, with corresponding eigenfunctions $u_{1}(n), \ldots, u_{s+l+m+1}(n)$.

Also, if $\lambda_{0}=0, \lambda_{1}, \ldots, \lambda_{s+l+m}$ are the eigenvalues of any one of the original boundary value problems (10)-(12), in Theorem 4.2, with corresponding eigenfunctions $z(n)$, $u_{1}(n), \ldots, u_{s+l+m}(n)$, then $\lambda_{1}, \ldots, \lambda_{s+l+m}$ are the eigenvalues of the corresponding transformed boundary value problems (10)-(12), in Theorem 4.2, with corresponding eigenfunctions $u_{1}(n), \ldots, u_{s+l+m}(n)$.

Proof By Theorems 2.1, 3.2, 3.3, 3.4 we have that (2.1) transforms eigenfunctions of the original boundary value problems (1)-(9) to eigenfunctions of the corresponding transformed boundary value problems. In particular, if $\lambda_{1}, \ldots, \lambda_{s+l+m+1}$ are the eigenvalues of one of the original boundary value problems, (1)-(9), with eigenfunctions $u_{1}(n), \ldots, u_{s+l+m+1}(n)$, then:

(i) $\frac{1}{z(n-1) c(n-1)}, u_{1}(n), \ldots, u_{s+l+m+1}(n)$ are the eigenfunctions of the corresponding transformed boundary value problem, (1)-(3), with eigenvalues $\lambda_{0}=0, \lambda_{1}, \ldots, \lambda_{s+l+m+1}$. Since the transformed boundary value problems, (1)-(3), have $s+l+m+2$ eigenvalues, it 
follows that $\lambda_{0}=0, \lambda_{1}, \ldots, \lambda_{s+l+m+1}$ constitute all the eigenvalues of the transformed boundary value problem;

(ii) $u_{1}(n), \ldots, u_{s+l+m+1}(n)$ are the eigenfunctions of the corresponding transformed boundary value problem, (4)-(9), with eigenvalues $\lambda_{1}, \ldots, \lambda_{s+l+m+1}$. Since the transformed boundary value problems, (4)-(9), have $s+l+m+1$ eigenvalues, it follows that $\lambda_{1}, \ldots, \lambda_{s+l+m+1}$ constitute all the eigenvalues of the transformed boundary value problem.

Also, again by Theorems 2.1, 3.2, 3.3, 3.4 we have that (2.1) transforms eigenfunctions of the original boundary value problems (10)-(12) to eigenfunctions of the corresponding transformed boundary value problems. In particular, if $\lambda_{0}=0, \lambda_{1}, \ldots, \lambda_{s+l+m}$ are the eigenvalues of one of the original boundary value problems, (10)-(12), with eigenfunctions $z(n)$, $u_{1}(n), \ldots, u_{s+l+m}(n)$, then $u_{1}(n), \ldots, u_{s+l+m}(n)$ are the eigenfunctions of the corresponding transformed boundary value problem, (10)-(12), with eigenvalues $\lambda_{1}, \ldots, \lambda_{s+l+m}$. Since the transformed boundary value problems, (10)-(12), have $s+l+m$ eigenvalues, it follows that $\lambda_{1}, \ldots, \lambda_{s+l+m}$ constitute all the eigenvalues of the transformed boundary value problem.

\section{Competing interests}

The authors declare that they have no competing interests.

Authors' contributions

Both SC and ADL worked jointly and separately on all aspects of this research.

Acknowledgements

SC was supported by NRF grant no. IFR2011040100017.

Received: 25 May 2012 Accepted: 30 May 2012 Published: 28 June 2012

doi:10.1186/1687-2770-2012-66

Cite this article as: Currie and Love: Erratum to: Hierarchies of difference boundary value problems. Boundary Value Problems 2012 2012:66.

\section{Submit your manuscript to a SpringerOpen ${ }^{\circ}$ journal and benefit from:}

- Convenient online submission

Rigorous peer review

- Immediate publication on acceptance

- Open access: articles freely available online

- High visibility within the field

- Retaining the copyright to your article 